\title{
Analytical STEM-in-SEM: Towards Rigorous Quantitative Imaging
}

\author{
Jason Holm ${ }^{1}$
}

1. Applied Chemical and Materials Division, National Institute of Standards and Technology, Boulder, United States.

Modern solid-state transmission electron detectors and conventional scanning electron microscopes are widely available and easy to use, making the suite of techniques referred to as Scanning Transmission Electron Microscopy in a Scanning Electron Microscope (STEM-in-SEM) more accessible today than ever before. These techniques are especially well-suited to a host of applications ranging from nanoparticle metrology [1], to biological or organic sample imaging [2-5], to orientation imaging microscopy [6, 7]. By using low energy primary electrons (i.e., $<30 \mathrm{keV}$ ) compared to higher energy primary electrons (i.e. $>80 \mathrm{keV}$ ), interaction between the incident electron beam and the sample is more probable, which ultimately means that more information can be obtained. Qualitative image interpretation and extracting quantitative information, however, is not always straightforward since multiple electron scattering events occur in the vast majority of samples. Moreover, rigorous quantitative image analyses can be challenging because electron scattering cross-sections at these energies are not especially well quantified, particularly for low atomic number elements. And, although STEM-in-SEM techniques have existed since the inception of the SEM, very few rigorous experimental transmission imaging studies at conventional SEM energies have been published [8-10].

In this contribution, a straightforward modular aperture system that can be adapted to most STEM detectors will be described [11]. It will be shown how this system can be used to make an angularly sensitive imaging system out of an otherwise rudimentary transmission detector. A rigorous application of that system to thin films comprising materials ranging from carbon to platinum will be demonstrated. From a practical perspective, an improved understanding of image contrast is possible with this system. From a more fundamental perspective, it may be possible to extract electron scattering cross-sections at low energies and for low atomic number elements.

To those ends, systematic experimental studies of several thin films will be presented. Effects of primary electron energy and transmission detector acceptance angle on STEM-in-SEM image intensities will be examined quantitatively and compared with results predicted by Monte Carlo-based electron scattering simulations. Angular distributions of forward scattered electrons will be quantified experimentally using a thin annular detector, ways to obtain imaging conditions that enable rigorous quantitative image interpretation will be described, and mass-thickness contrast at different primary electron energies and detector acceptance angles will be examined.

In one example, commercially available $\mathrm{Si}_{3} \mathrm{~N}_{4}$ films of different thickness $(10,20$, and $50 \mathrm{~nm})$ will be examined as a function of primary electron energy and transmission detector acceptance angle. Here, coherent elastic scattering is largely absent and mass-thickness contrast as a function of beam energy is addressed. Angular distributions of electrons forward scattered through the films are quantified and compared with Monte Carlo-based electron scattering simulations, and unanticipated peak splitting in the scattering distributions is observed. In another example, angular distributions of electrons forwardscattered through $\mathrm{C}, \mathrm{Ni}, \mathrm{Co}, \mathrm{Pd}$, and Pt films will be quantified (Figure 1). In addition to quantifying the effect of primary electron energy on the scattering distributions, the following questions will be 
addressed: Is there an optimal acceptance angle range for quantitative imaging? How easily can elements spanning the periodic table be discerned? Will Pt films always exhibit stronger contrast against vacuum levels than $\mathrm{C}$ films if both are the same nominal thickness? Can films of neighboring elements (i.e., $\mathrm{Ni}$ and $\mathrm{Co}$ ) and similar thickness be discerned?

References:

[1] E. Buhr et al, Meas. Sci. Technol. 20 (2009) 084025.

[2] M. Kuwajima et al, PLOS ONE 8 (2013) e59573.

[3] O. Guise et al, Polymer 52 (2011) 1278-1285.

[4] N. Hondow et al, Nanotoxicololgy 5 (2011) 215-227.

[5] C. A. Garcia-Negrete et al, Analyst 140 (2015) 3082-3089.

[6] R. Keller and R. Geiss, J. Microscopy 245 (2012) 245-251.

[7] P. Trimby et al, Acta Mater. 62 (2014) 69-80.

[8] V. Morandi and P. Merli, J. Appl. Phys. 101 (2007) 114917.

[9] T. Volkenand et al, Microsc. Microanal. 16 (2010) 604-613.

[10] M. Pfaff et al, J. Microsc. 243 (2011), 31-39.

[11] J. Holm and R. Keller, Ultramicroscopy 167 (2016) 43-56.

[12] This work is a contribution of the US Government and is not subject to copyright in the United States.
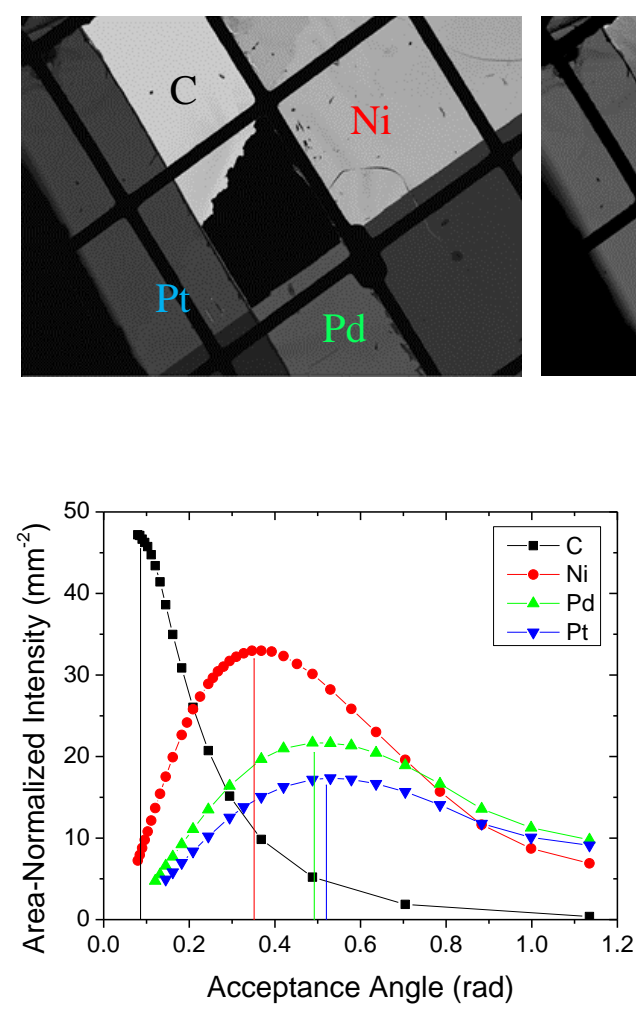

(b)
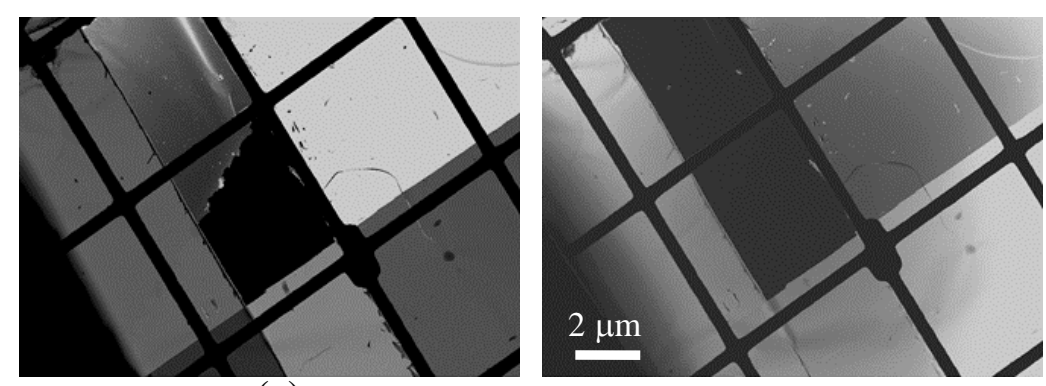

(a)

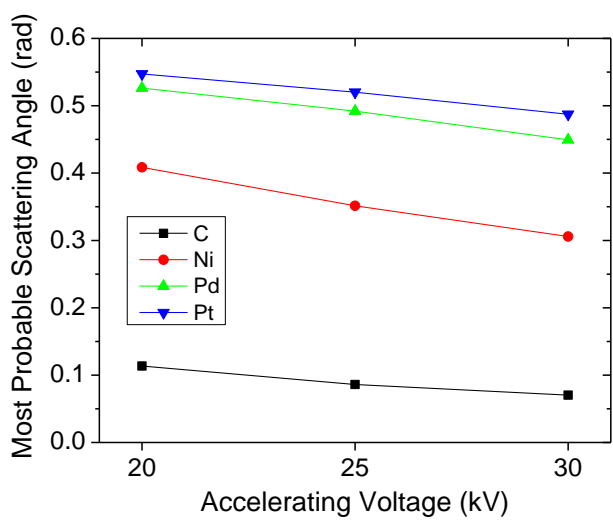

(c)

Figure 1. (a) Three STEM-in-SEM images of $~ 100 \mathrm{~nm}$ thick $\mathrm{C}, \mathrm{Ni}, \mathrm{Pd}$, and Pt films showing contrast changes with increasing detector acceptance angle (acceptance angle increases from left-to-right). (b) Image intensity distributions as a function of detector acceptance angle for $25 \mathrm{keV}$ primary electrons, and (c) A summary of the most probable scattering angles for the four films as a function of primary electron energy. 